\title{
MAS architecture and knowledge model for vehicles data communication
}

\author{
Emmanuel Adam, Emmanuelle Grislin-Le Strugeon, \\ René Mandiau
}

Lille Nord de France, F-59000 Lille, France

UVHC, LAMIH, F-59313 Valenciennes, France

CNRS, UMR 8201, F-59313 Valenciennes, France

KEYWORD

\section{Multiagent Systems}

Mobile Entities

Knowledge Communication

Traffic Simulation

\section{ABSTRACT}

Completely autonomous vehicles in traffic should allow to decrease the number of road accident victims greatly, and should allow gains in terms of performance and economy.

Modelling the vehicles interaction, and especially knowledge sharing, is one of the main challenges to optimize traffic flow with autonomous vehicles.

We propose in this paper a model of knowledge communication between mobile agents on a traffic network. The model of knowledge and of interaction enables to propagate new knowledge without overloading the system with a too large number of communications. For that, only the new knowledge is communicated, and two agents communicate the same knowledge only once. Moreover, in order to allow agents to update their knowledge (perceived or created), a notion of degradation is used.

A simulator has been built to evaluate the proposal, before to implement it in mobile robots. Some results of the simulator are proposed in this article.

\section{Introduction}

In many studies on traffic supervision, the optimization of traffic flow as well as new road infrastructure [BAZZAN, A.L. 2005], attempts to deal with collective interests and individual interests.

We think that completely autonomous vehicles in traffic should allow great decrease in the number of road accident victims, and should allow gains in terms of performance and economy. Developing models of the interaction among the different vehicles is one of the main challenges to optimize traffic flow with autonomous vehicles [DRESNER \& STONE 2008].

The simulation of traffic is often used to evaluate traffic flow optimization methods.

In the area of road traffic simulation, two approaches allow management policies for scheduling vehicles flows: centralized approaches and distributed approaches (behavioural approaches).

In centralized approaches for traffic simulation, the first traffic flow models were mathematical. Such a model allows, for instance, a highway traffic situation to be modelled using car-following laws, which are, in fact, differential equations that are obtained empirically through regression using data collected at currently operating road sections [LIEBERMAN \& RATHI 1997]. Even now, most of the microscopic simulations use the car-following law to model inline driving, while the specific case of the intersection is managed using centralized scheduling techniques. In these applications, each vehicle approaching the intersection is placed in a virtual queue - one for each branch of the intersection. This search process is called "gap acceptance". For instance, Vissim [VISSIM 2005] has a "yellow box" parameter, which 
allows users to define a minimal speed that the vehicles inside the intersection must respect so that other vehicles can enter the intersection. These traffic simulation tools assume that a centralized scheduler makes decisions for each vehicle and lets enter the intersection only when their trajectories are not in conflict. The simulated behaviours of individual drivers produced by schedulers are not always realistic, and thus many traffic phenomena can not be simulated (e.g., traffic signals violation and traffic congestion inside the intersection).

The behavioural approach considers traffic as an emerging phenomena resulting from actions and interactions of the various traffic system actors (e.g., car drivers, pedestrians, road operators). The behavioural approach aims to accurately model and reproduce the behaviours and interactions of the simulated entities in order to obtain realistic traffic phenomena. The realism of the emergent traffic also depends on the distribution of behaviours among the set of simulated entities (i.e., heterogeneity of the individual practices). Different models have been developed for building this traffic: cellular automata approaches [RUSKIN \& WANG, 2002], robotic inspired approaches [REECE \& SHAFER, 1993], multi-agent approaches [BAZZAN, 2005, 2010; DRESNER \& STONE, 2008; KETENCI et al. 2010; VASIRANI \& OSSOWSKI, 2011].

The principal advantage of this behavioural model is that simulation conditions can be dynamically modified (the degree of visibility which results from the weather, the driving preferences of the human driver, the characteristics of the autonomous vehicle - cars, lorries, buses, pedestrians etc.) as can the road equipment (traffic signals, traffic signs, etc.).

So, in the context of a project that aims at studying the impact of information communication between drivers and infrastructure or elements of the environment (like shops, car park for instance), we turn to multiagent systems to propose a simulator of a traffic network.

The next section of the paper presents a synthetic state of the art relative to the problem of trust inherent to the communication of knowledge between autonomous entities.
Next, our architecture of the multiagent system used to share knowledge between mobile agents ${ }^{1}$ proposed.

The section 4 presents the simulator of road traffic, and the case study that we used to validate our proposal.

The last section draws our conclusions and gives some perspectives for future research.

\section{Knowledge communica- tion between autonomous en- tities}

Communication of knowledge implies classically to take care of the confidence about this knowledge.

If an agent $a$ that has a current knowledge set $\beta_{a}$ receives a new knowledge $b$, this new knowledge can be:

- from a doubtful origin: the knowledge comes from an unknown agent, or without the required signature. In this case, it is necessary to exchange with a standard (like FIPAACL) communication language. The use of ontology can give a kind of guarantee that the sender is a "good agent". If necessary, the use of a particular signature could avoid "hacker agent" to send information.

- $\quad$ inconsistent: the receiver of the knowledge $b$ is unable to store it in its own knowledge without getting an inconsistency; $(\{b\} \cup$ $\left.\beta_{a}=\varnothing\right)$. This inconsistency can lead either to the reject of $b$, or a revision of $\beta_{a}$ so that it accepts $b$, or a categorization of the sender as a "suspicious agent".

- out-of-date: dynamic environment implies to date the knowledge about it, about events. When the knowledge $b$ is received by an agent, if it deals with an element $b_{a}$ stored in this agent, this latter has to check if the date of the received knowledge is newer than its own knowledge before accepted it.

About the doubtful origin of the information and more generally, the security in MAS, the recent sur-

\footnotetext{
1 In the following, "mobile agents" mean agents that represent mobile entities (and not especially mobile code).
} 
vey in [JUNG, Y. et al. 2012] gives details on the access control and trust/reputation existing solutions. In centralized models, trust and reputation measures result from aggregating rankings about the information sources, in a discrete form as what is proposed to users by e-commerce sites, or in the form of a probability expectation value (see the approaches reviewed in [JOSANG, A. et al. 2007]). In distributed systems, reputation and trust are subjective properties [PINYOL, I. \& SABATER-MIR, J. 2011]: each agent builds its own representation of the others' credibility. For our application to traffic simulation, we will assume that the agents are cooperative and well functioning.

About the knowledge inconsistency, the problem is to corroborate information in checking them against multiple sources. Regarding this issue, many studies are currently realized in the two domains of the wireless sensor data [KHALEGHI, B. et al. 2011] and the Web information fusion and integration [YAO, J. et al. 2008]. Knowledge consistency is a pertinent problem in the traffic context, but not the central point of our current study.

In a first attempt, our work is focused on the outof-date problem that concerns the time-dependent reliability of the knowledge. The problem of the out-ofdate data is tackled in the information system domain as the information freshness [BOUZEGHOUB, M. 2004]. Information freshness describes how old is the received data and/or their currency with respect to their sources. It is a key factor for the information quality, especially in the context of distributed systems, composed of autonomous data sources. One of the approaches that can contribute to the information quality is time stamping. However, unlike the methods in which timestamp is used to ensure the quality of the sources, e.g. [JATOWT, A. et al. 2011], we use timestamp at a more detailed level, to ensure the quality of every data exchanged.

\section{Model of communication between mobile agents in a network}

Fast communication of knowledge between mobile agents along a network can be done: directly, by messages exchange, when agent are physically close enough to communicate; or indirectly through the environment (generally the nodes are used to store / read information). We think that a node should be more pro-active and should have the opportunity to choose to communicate some information to some agents chosen according to its knowledge. So we propose a third method in which some non mobile agents are located at the nodes and communicate with mobile agents that are close enough to receive messages. Moreover, we can easily argue that to give to the nodes the possibility to interact with one another would ameliorate efficiency to the propagation of information along the network.

\section{Multilevel architecture.}

In order to allow the three kinds of communication ('mobile-agent-to-near-mobile-agent', 'mobileagent-to-near-node' and 'node-to-near-node'), and to allow communication between distant mobile agents and distant nodes, we use a multi-level architecture, inspired from holonic principles.

We have already used this kind of architecture for the simulation of a flexible assembly cell, in order to correct myopic behaviour of mobile and autonomous shuttle [ADAM et al. 2011].

At the bottom of the system (level 0), we have the mobile agents. At the level 1, there are the node agents, which can schedule, manage conflicts between 'mobile agents' that have to cross them. These agents (mobiles and nodes) and the environment (the network) are included in the 'network agent' that represents the multiagent system (cf. Fig. 1.a).

Including 'node agents' leads to two possible organisations depending on the activity of these agents:

- 'node agents' have only a communicating behaviour, so they are at the same level as 'mobile agents' (cf. Fig. 1.b)

- 'node agents' can schedule, manage conflicts between 'mobile agents' that have to cross them. (cf. Fig. 1.c).

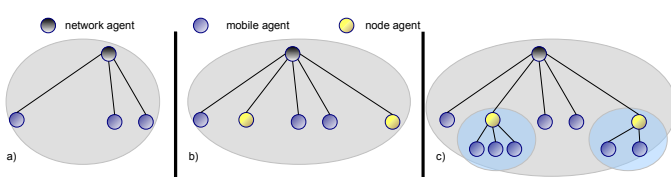

Fig. 1 Organisations of mobile agents on a network

The communications between agents do not really follow the holonic rules that impose communications between agents of a same layer or between an agent 
and its responsible. Indeed, in our case each agent can interact with all agents that are in its 'vision field'; therefore a communication between a mobile agent and another mobile agent that is enclosed in a node agent is possible.

The Figure 2 presents an extract from a screenshot of a traffic simulator that we use to test knowledge communication between cars; 'mobile agents' are represented by 'vehicle agents', 'node agents' are represented by 'crossroad managers'.

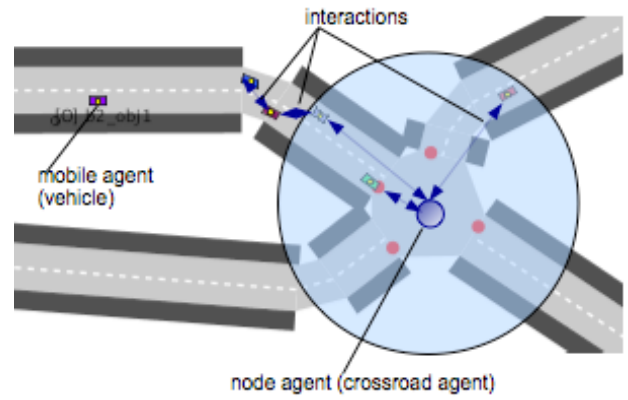

Fig. 2. Example of application in road traffic. Mobile vehicles agents interact between others and with the crossroad (node) agent if they are close enough.

\section{Elements of knowledge model.}

We give here just some elements of how the mobile agents manage the exchanged knowledge. A knowledge is a partial view of the environment or of the other agents, namely for a given object o of the environment (the traffic network for example); it is (generally) an incomplete copy of it, so it is a representation of o with missing attributes and methods.

If $\mathcal{O}$ is the set of objects of the environment, an object $\mathrm{o}$ is defined by its identity, its attributes and functions:

$$
\mathrm{o}=\left(\begin{array}{c}
i d, \\
\text { attributes }_{\mathrm{o}}=\left\{a_{1}^{o}, \ldots, a_{n_{\mathrm{n}>0}}^{o},\right. \\
\text { functions }_{\mathrm{o}}=\left\{f_{1}^{o}, \ldots, f_{m}^{o}\right\}_{\mathrm{m} \geq 0}
\end{array}\right)
$$

If $o_{a}^{\prime}$ is a partial representation of $o$ for the agent $a$, the cardinalities of the set of attributes and functions of $o_{a}^{\prime}$ are lesser than those of the object $o$ :

and

$$
\mid \text { attributes }_{o^{\prime}}|\leq| \text { attributes }_{o} \mid
$$

$$
\mid \text { functions }_{o^{\prime}}|\leq| \text { functions } s_{o} \mid
$$

We define a knowledge $\kappa_{o}^{a}$ (cf. def. 2) on an object $o$ for an agent $a$ by:
- $\quad o_{a}^{\prime}$, a partial view of $o$ from $a$;

- date $_{\kappa_{o}}$, the date when the knowledge has been created or updated (by $a$ or by another agent if the knowledge has been received);

- builderAgent ${ }_{\kappa_{o}}$, the 'builder' of the knowledge (name of the agent that has created/updated the knowledge from its perception);

- senderAgent $\kappa_{\kappa_{o}}$, the 'sender' of the knowledge (name of the agent that could have sent the knowledge to $a$ );

- receiverAgents $\kappa_{o}^{a}$, the list of agents to whom the knowledge has been sent by $a$;

- shareable $_{\kappa_{o}^{a}}$, the fact that the knowledge is shareable or not by $a$.

$\kappa_{o}^{a}=\left(\begin{array}{c}o_{a}^{\prime}, \text { date }_{\kappa_{o}}, \text { builderAgent }_{\kappa_{o}}, \text { senderAgent }_{\kappa_{o}} \\ \text { receiverAgents }_{\kappa_{o}^{a}}, \text { shareable }_{\kappa_{o}^{a}}\end{array}\right)(2)$

The notions of date ${ }_{\kappa_{\mathrm{o}}}$ and of receiverAgents ${ }_{\kappa_{\mathrm{o}}^{\mathrm{a}}}$ allow to decrease the number of communications. Indeed, when the agent $a$ perceives, from its perception function, an image of a new object $o$, it creates the knowledge $\kappa_{o}^{a}$, stamped with the date of creation.

When the agent $a$ receives from another agent $b$ a knowledge on the object $o, a$ replaces its knowledge only if the date $\kappa_{\kappa_{o}}$ in $\kappa_{o}^{b}$ is newer than the one in $\kappa_{o}^{a}$.

If $b$ is close enough to send its knowledge to $a$, reciprocally $a$ can send its knowledge to $b$. Their interaction is used to create a common representation based on knowledge sharing. The most recent knowledge on the object $o$ is preferred to any previous knowledge on the same object, no matter which is the information sender (no notion of trust here). As a consequence, when two agents meet, at the end of the communication, they own the last known representation of the object $o$.

When an agent $(a)$ sends some knowledge on $o$ to another $(b)$, it adds this agent to its set of receivers (receiverAgents $s_{\kappa_{o}^{a}} \leftarrow$ receiverAgents $s_{\kappa_{o}^{a}} \cup\{b\}$ ).

Each time an agent receives a new version of an information, its set of agents to which this information has been sent is emptied (receiverAgents $\kappa_{\kappa_{\sigma}^{a}} \leftarrow$ \{\}$)$.

This allows to decrease the number of communications; for instance, when $a$ and $b$ meet many times (for example, if they follow the same path), the communication about a given knowledge is done only once. 


\section{Simulation of knowledge communication in a traffic}

We illustrate our proposal on a traffic road simulator that we have developed in the context of a project (Plaiimob : a simulating Plateform dedicated to Mobility services) of CISIT (for International Campus on Security and Inter modality in Transports).

The aim of this project is to allow vehicle-tovehicle $(\mathrm{V} 2 \mathrm{~V})$ communication to allows drivers to automatically exchange data about their environment (incident/traffic jam on a road, information about offstreet parking ...) [POPOVICI, D. et al. 2011].

We developed a traffic road simulator with the Jade Platform ${ }^{2}$ :

- The environment is a traffic network in the OpenStreetMap format ${ }^{3}$ (OSM).

This allows us to use true traffic networks, or to define our own maps in order to test particular situations.

- The classes of agents are: the PersonnageAI agent class (it allows to simulate the behaviour of a driver), the CrossRoadManager agent class (it allows to manage the priorities at a crossroad according to the road signs), the ObserverAgent class (that allows to draw statistics from the simulating exercise).

- $\quad$ The roles played by the PersonnageAI are: RandomBehaviourRole: to represent a driver that moves randomly on the traffic; BusRole: to represent a driver that starts from a particular point and has to reach an objective, by linking some bus stops, at earliest; EmergencyRole: to represent a driver of an ambulance, firetruck, for instance, that has an objective to reach as soon as possible.

- $\quad$ The roles played by the CrossRoad Managers are linked to the road signs or traffic

\footnotetext{
${ }^{2}$ See the web site of the Jade platform:

http://jade.tilab.com/

${ }^{3}$ OpenStreetMap is a collaborative project that allows to edit free maps of the world; with free tools, it is possible to use maps of a particular region/town/part of town in order to simulate traffic on a representation of the real world. See the web site of the OpenStreetMap project:

http://www.openstreetmap.org/
}

lights associated with them. We defined some roles like: FifoCrossRoad (the first vehicle arrived in a queue of the crossroad 'receives' a green light); ClassicTrafficLightRole (emulation of classical traffic lights at each entry of the crossroad); AITrafficLightRole (based on the ClassicTrafficLightRole, it gives the priority to the street from which an emergency vehicle arrives (the associated traffic light goes on green light) ...).

Jade has been chosen because the aim of the project is to test our proposal on a real case, with agents embedded in the smart-phones of the drivers.

So we aim at reusing some classes of the simulation to build agents and their roles.

\section{Communication of incident.}

In order to test the benefit to have a V2V communication, we present here a small case study that includes 2 bus lines $(A$ and $B$ ), with one bus for the line $A$ (busla) and 5 bus of the line $B$ (bus $2 a, \ldots, b u s 2 e)$; and five cars that travel randomly on the network (randomCarl, .., randomCar5).

The Fig. 3 shows the map used in this context. The bus of the line $A$ starts from 'SA', and has to make loops between the bus stops 'BS-A1' and 'BS$\mathrm{A} 2$ '. For the buses of the line $B$, they start from 'SB', and have to make loops between the bus stops 'BS$\mathrm{B} 1$ ', and 'BS-B2'. 'SR' is the starting point of the cars that travel randomly.

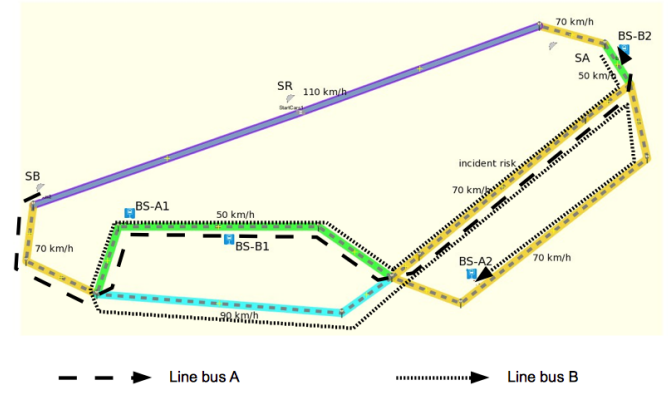

Fig. 3. Map of Traffic Roads used in the case study.

The buses have to stop on the right side of the road. One road is prone to incident risk

We used three different scenarios. In the first scenario, there is no incident; all the vehicles travel as they planed. In the second scenario, we add an incident on the road with incident risk before busla 
reaches it, and vehicles do not communicate information. In the third scenario, buses and cars are able to exchange knowledge. The incident causes a tailback on this road, and the speed limit is divided by 2 (so it becomes $35 \mathrm{~km} / \mathrm{h}$ maximum).

Initially, each mobile agent knows the map of the traffic road, with the initial speed limit.

Each time a mobile agent reaches a road, it updates its knowledge if needed: if the perceived state of the road implies a modification of the speed limit (due to an incident, or traffic jam, for example), a knowledge is created and stored; if the perceived road exists in the knowledge, the knowledge is updated (the date is updated, the state of the road is modified if necessary).

If no incident is signalled, but if the agent took at least twice more time to exit the road than what it predicted, it records a tailback event in its knowledge. Each 'PersonnageAI' with the role 'BusRole' is able to compute the shortest path to its next objective with the Dijkstra algorithm [DIJKSTRA, E.W. 1959]. The best path, without incident, for A is to make a double loop (a ' 8 ') : from 'SA', the bus goes by the road with the 'incident risk'; then to 'BS-A1'; then it takes the road limited to $90 \mathrm{~km} / \mathrm{h}$; takes again the 'incident risk' road; to reach 'BS-A2' and repeats its round.

For the buses of the line $\mathrm{B}$, the best path starts from 'SB'; goes to 'BS-A1'; takes the 'incident risk' road to reach 'BS-A2'. The buses take next the fast lane to repeat their round.

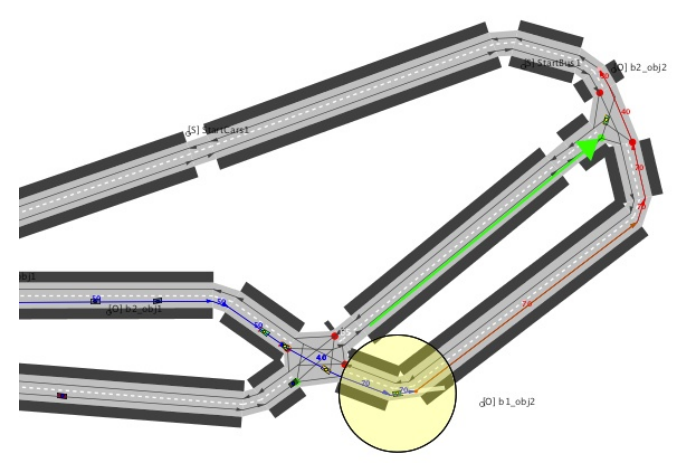

Fig. 4. Screencopy of the Traffic Roads Simulator. bus $2 b$ is selected;

the red arrow shows the roads where it plans to go.

The disc around bus $2 b$ represents its communicating zone.

In scenario 3, we choose to put an incident at the beginning of the simulation; busla, which does not know this fact, travels on the route with incident. It informs all the agents that it meets during its move. bus $2 b$ is the first informed by busla, it computes a new best path and chooses another road than the one taken by bus $2 a$.

On Fig. 4, the green arrow shows the road took by bus $2 a$, the red arrow shows the road took by $\operatorname{bus} 2 b$ (and its followers) that met busla.

Without communication between vehicles, the lost time when an incident occurs on the 'incident risk' road is 7 time units on average. The communication of information, in the particular case study, results in a timesaving of about 4 time units.

The table 1 shows the results issued from the three scenarios. Without communication between vehicles, the lost time when an incident occurs on the 'incident risk' road is 7 time units on average. The communications of information, in the particular case study, result in a timesaving of about 4 time units.

\begin{tabular}{|l|l|l|l|}
\hline Classes & Scenario 1 & Scenario 2 & Scenario 3 \\
\hline bus1a & $1: 31 \mathrm{tu}$ & $1: 38 t u$ & $1: 38 t u$ \\
\hline bus2a & $0: 48 t u$ & $0: 57 t u$ & $0: 56 t u$ \\
\hline bus2b & $0: 50 t u$ & $0: 56 t u$ & $0: 52 t u^{4}$ \\
\hline bus2c & $0: 50 t u$ & $0: 56 t u$ & $0: 53 t u^{5}$ \\
\hline bus2d & $0: 51 t u$ & $0: 55 t u$ & $0: 52 t u^{5}$ \\
\hline bus2e & $0: 48 t u$ & $0: 56 t u$ & $0: 51 t u^{5}$ \\
\hline
\end{tabular}

Table. 1. Result issued from simulation of the three scenario (in time units (tu))

\section{Communication of incident repairing.}

In the simulator, when a incident is removed from a road, this one goes back to its initial speed limit. If all the buses have got the knowledge that an incident has occurred on the road, they will never take this road again. They could get the information about the repairing only if a vehicle with a RandomBehaviourRole takes this road and meets the buses.

Results from the simulation about this propagation of incident repairing are not really pertinent, because it depends on the random choice of path by the vehicles.

So if a simulation does not include 'random cars', once a path is declared 'with incident', and once the

\footnotetext{
${ }^{4}$ bus $2 \mathrm{a}$ arrives on the 'incident risk' zone before meeting bus 1 a.

bus $2 b, \ldots$, bus 2 e receive a new knowledge from bus $1 \mathrm{a}$ (incident on the road) and recompute a best path to join 'BS-B2'.
} 
event has been propagated to all mobile agents, the path is neither reused. It is important to allow agents to be informed about a repairing; so it is important to allow at least one mobile agent to perceive this repairing. A mobile agent, having the information that an incident has occurred on a path, has to decide to take it, after some time.

We introduce a notion of degradation, in the definition of a knowledge $\kappa_{o}^{a}$. We define degradation $_{\kappa_{o}}$ as a coefficient of the reliability of the knowledge (if $t$ is the elapsed time since the creation/update of the knowledge, the confidence that a has on it is: trust_ $\kappa_{o}=1-t \times$ degradation $_{\kappa_{o}}$ ). For a knowledge $\kappa_{o}$, if trust $_{\kappa_{o}} \leq 0$, then the agent does not take into account this knowledge to compute the best path to its next objective; and the knowledge is not sent to other agents.

If degradation $\kappa_{\kappa_{o}}=0$, then the knowledge $\kappa_{o}$ is perennial; if a degradation $\kappa_{\kappa_{0}}=0.1$ then the knowledge $\kappa_{o}$ exists for 10 tu (time-unit).

The degradation coefficient for the knowledge is strongly dependent of the case study. Here, due to the light complexity of the traffic network used, as a bus needs $2.2 t u$ to make a loop, we fix the degradation coefficient of the knowledge about an incident at $1 / 3$ $t u$. This allows a bus to try to pass by the incident road' after 3 loops; if the incident is always on the road, the date of the knowledge is updated, and the new knowledge is communicated to each vehicle met by the bus.

One perspective of this work could be to automatically define the degradation coefficient from observation during the simulation.

\section{Propagation of knowledge.}

In order to improve the diffusion of knowledge, we propose to use agents linked to the nodes of the network (here, these agents are the CrossRoad Managers). The organisation of the MAS follows the one presented in (cf. Fig. 1.c).

When a mobile agent decides to communicate its knowledge, it includes the local node agents in its potential recipients. All nodes are not necessary equipped with node agents, but in the case study, a CrossRoad agent is located to each crossroad. When a vehicle detects a problem on the road it reaches, the vehicle informs all the agents inside its perimeter of communication. The crossroads at the beginning and the end of the road receive the information. When another vehicle approaches the road, the crossroad where the vehicle arrives communicates the knowledge on the road incident/repairing. The vehicle is able to recompute its best path, and to choose another road.

We defined thus a fourth scenario with crossroad manager agents able to communicate knowledge. The results of the simulation is the same as for the scenario 3, except that all the line $B$ buses, even the first, 'bus $2 a$ ', are informed about the incident. Indeed, when the Line A bus 'busla' detects the incident, it sends this knowledge to each crossroad agent it meets. These ones send the knowledge to the others crossroad manager agents linked to them (as said previously, an information can be received several times by an agent, from different senders but it is stored only once and sent only once to a particular recipient).

When the first bus of line $B$ arrives at the entry of the damaged road, it is already aware of that fact and is able to compute a best path to reach its goal by avoiding the road.

The communication of knowledge through crossroad managers is very interesting, regarding the communication of incident repairing, when vehicles with the RandomBehaviourRole are used in the simulation. Indeed, the information is propagated immediately once a first vehicle takes the road by chance. Without the presence of such vehicles in the simulating exercise, the benefit of having communicating crossroad managers is not relevant, regarding the communication of incident repairing, because the knowledge is degraded relatively in the same way in all mobile agents.

We plan to enhance the information sent by the crossroad agents: when a car informs a crossroad agent about its desire to take a particular road, the crossroad manager sends the number of agents that have already taken this road in a near past. So the mobile agent can choose to pursue its initial plan, or to recompute a new best path.

\section{Conclusion}

In order to allow the propagation of knowledge between mobile agents, with a minimum of exchanged messages, we propose an architecture, a model of knowledge and a model of communication. 
In the first results presented in this paper, we make the assumption that all the agents are cooperative, and no deficient; that is to say that they cannot send wrong knowledge, voluntary, or not (if a sensor has a dysfunction).

In our current approach, the trust on a knowledge depends only on the date from which the knowledge has been updated or created. We plan to introduce the notion of trust that depends of the sender; for example, if an agent sends a knowledge that is not coherent with the information from other agents, these latters can decide to put in quarantine the faulty agent, like in [VERCOUTER \& JAMONT, 2011] for example. In order to decide to integrate new knowledge about object $o$, an agent should, at first, collect data from multiple sources, to be able then to evaluate their coherence. This would decrease the risk due to dysfunctional or malicious agents, but would increase the de- lay before the agents take into account the occurrence of an incident in the traffic. This is, we think, a interesting issue when mobile agents evolve in a dynamic environment like traffic road.

\section{Acknowledgment}

This research was financed by the International Campus on Safety and Inter-modality in Transportation, the Nord/Pas-de-Calais Region, the European Community, the French Regional Delegation for Research and Technology, and the French National Centre for Scientific Research. We are grateful for the support of these institutions. The authors thank particularly Remy Tylski, who implemented interesting parts of the simulator.

\section{References}

[ADAM, E. et al. 2011]

[BAZZAN, A.L. 2005]

[BAZZAN, A.L. et al. 2010]

[BOUZEGHOUB, M. 2004]

[DIJKSTRA, E.W. 1959]

[DRESNER \& STONE, 2008]

[FERBER, J. 1999]

[JATOWT, A. et al. 2011]

[JENNINGS, N.R. et al. 1998]
ADAM, E, ZAMBRANO, G., PACH, C., BERGER, T., TRENTESAUX, D. Myopic behaviour in holonic multiagent systems for distributed control of FMS. In: J. Corchado, J. Pérez, K. Hallenborg, P. Golinska, R. Corchuelo (eds.) Trends in Practical Applications of Agents and Multiagent Systems, Advances in Intelligent and Soft Computing 90,91-98. Springer, 2011.

BAZZAN, A.L. A distributed approach for coordination of traffic signal agents. Autonomous Agents and Multi-Agent Systems, 10, 131-164, 2005.

BAZZAN A.L.C., DE OLIVEIRA D., and C. DA SILVA B. Learning in groups of traffic signals. Engineering Applications of Artificial Intelligence, 23(4):560 $568,2010$.

BOUZEGHOUB, Mokrane. A framework for analysis of data freshness. In Proc. of the 2004 international workshop on Information quality in information systems (IQIS '04). ACM, New York, NY, USA, 59-67, 2004.

DIJKSTRA, E.W. A note on two problems in connexion with graphs. Numerische Mathematik 1, 269-271, 1959.

DRESNER,K., STONE, P. Mitigating catastrophic failure at intersections of autonomous vehicles. In: AAMAS Workshop on Agents in Traffic and Transportation, 78-85. Estoril, Portugal, 2008.

FERBER,J. Multi-agent systems - an introduction to distributed artificial intelligence. Addison- Wesley-Longman, 1999.

JATOWT, Adam, KAWAI, Yukiko, TANAKA, Katsumi. Calculating content recency based on timestamped and non-timestamped sources for supporting page quality estimation. In Proceedings of the 2011 ACM Symposium on Applied Computing (SAC '11). ACM, New York, NY, USA, 1151-1158, 2011.

JENNINGS, N.R., SYCARA, K., WOOLDRIDGE, M. A roadmap of agent research and development. Int. Journal of Autonomous Agents and Multi-Agent 
[JOSANG, A. et al. 2007]

[JUNG, Y.et al. 2012]

[KETENCI, U-G. et al. 2010]

[KHALEGHI, B. et al. 2011]

[LIEBERMAN \& RATHI, 1997]

[MANDIAU, R. et al. 2008]

[PINYOL, I. \& SABATERMIR, J. 2011]

[POPOVICI, D. et al. 2011]

[REECE \& SHAFER, 1993]

[RUSKIN \& WANG, 2002]

[VASIRANI \& OSSOWSKI, 2011]

[VERCOUTER \& JAMONT, 2011]

[VISSIM, 2005]

[YAO, J. et al. 2008]
Systems 1(1), 7-38, 1998.

JØSANG, Audun, ISMAIL, Roslan, BOYD, Colin. A survey of trust and reputation systems for online service provision. Decision Support Systems, 43(2), 618644, 2007.

JUNG, Youna, KIM, Minsoo, MASOUMZADEH, Amirreza, JOSHI, James B. D. A survey of security issue in multi-agent systems. Artificial Intelligence Review, 37, 239-260, 2012.

KETENCI U-G., AUBERLET J-M., BRÉMOND R., GRISLIN-LE STRUGEON E. (2010). Impact of attentional factors in a multi-agent traffic simulation. Proc. of the 23rd Annual Conference on Computer Animation and Social Agents, CASA 2010, Saint-Malo, France, 2010.

KHALEGHI, Bahador, KHAMIS, Alaa, KARRAY, Fakhreddine O., RAZAVI, Saiedeh N. Multisensor data fusion: A review of the state-of-the-art, Information Fusion, in Press, 2011.

LIEBERMAN, E., RATHI, A. Traffic flow theory. Oak Ridge National Laboratory, Chapter Traffic simulation, 1997.

MANDIAU, R., CHAMPION, A., AUBERLET, J.M., ESPIE, S., KOLSKI, C. Behaviour based on decision matrices for a coordination between agents in a urban traffic simulation. Appl. Intell. 28(2), 121-138, 2008.

PINYOL, Isaac, SABATER-MIR, Jordi. Computational trust and reputation models for open multi-agent systems: a review, Artificial Intelligence Review, 1-25, 2011.

POPOVICI, D., DESERTOT, M., LECOMTE, S., PEON, N. Context-aware transportation services (CATS) framework for mobile environments. Int. Journal of Next-Generation Computing 2(1), 2011.

REECE, D. A., SHAFER, S. A. A computational model of driving for autonomous vehicles. Transportation Research 27 (1), 23-50, 1993.

RUSKIN, H.J., WANG, R. Modeling traffic flow at an urban unsignalized intersection. In Proc. of the Int. Conf. on Computational Science-Part I, ICCS '02, 381-390. Springer-Verlag, London, UK, 2002.

VASIRANI M. and OSSOWSKI S. A computational market for distributed control of urban

road traffic systems. IEEE TRANSACTIONS on Intelligent Transportation Systems, 12(2):313-321, June 2011.

VERCOUTER, L., JAMONT, J.P. Lightweight trusted routing for wireless sensor networks. In:Y.Demazeau, M. Pechoucek, J.M. Corchado, J.B. Pérez (eds.) PAAMS, Advances in Intelligent and Soft Computing 88, 87-96. Springer, 2011. Vissim 4.10. User Manual. Technical Report. PTV Planung Transport Verkehr AG:Karlsruhe, Germany, 2005.

YAO, JingTao, RAGHAVAN, Vijay V., WU, Zonghuan. Web information fusion: A review of the state of the art, Information Fusion, 9(4), 446-449, 2008. 